\title{
New formulas for the linearization coefficients of some nonsymmetric Jacobi polynomials
}

\section{Waleed M Abd-Elhameed ${ }^{*}$}

\begin{abstract}
The main aim of this paper is to develop four innovative linearization formulas for some nonsymmetric Jacobi polynomials. This means that we find the coefficients of the products of Jacobi polynomials of certain parameters. In general, these coefficients are expressed in terms of certain hypergeometric functions of the unit argument. We employ some symbolic algebraic computations such as the algorithms of Zeilberger, Petkovsek and van Hoeij for reducing such coefficients. Moreover, and based on a certain Whipple transformation, two new closed formulas for summing certain terminating hypergeometric functions of the unit argument are deduced. New formulas for some definite integrals are given with the aid of the derived linearization formulas.
\end{abstract}

MSC: 42C10; 33A50; 33C25; 33D45

Keywords: linearization coefficients; hypergeometric functions; Jacobi polynomials; symbolic computation; recurrence relations

\section{Introduction}

The general linearization problem consists of finding the coefficients $G_{i, j, k}$ in the expansion of the product of two polynomials $A_{i}(x)$ and $B_{j}(x)$ in terms of an arbitrary sequence of orthogonal polynomials $\left\{P_{k}(x)\right\}$, that is,

$$
A_{i}(x) B_{j}(x)=\sum_{k=0}^{i+j} G_{i, j, k} P_{k}(x) .
$$

An important particular case of problem (1) is the standard linearization or ClebschGordan-type problem, which consists of finding the coefficients $L_{i, j, k}$ in the expansion of the product of two polynomials $A_{i}(x)$ and $A_{j}(x)$ in terms of the sequence $\left\{A_{k}(x)\right\}_{k \geq 0}$, i.e.,

$$
A_{i}(x) A_{j}(x)=\sum_{k=0}^{i+j} L_{i, j, k} A_{k}(x) .
$$

Linearization problems appear in several applications. For example, the special case in which $i=j$ in the standard linearization formula (2) is very useful when evaluating the

(c) 2015 Abd-Elhameed. This article is distributed under the terms of the Creative Commons Attribution 4.0 International License (http://creativecommons.org/licenses/by/4.0/), which permits unrestricted use, distribution, and reproduction in any medium, provided you give appropriate credit to the original author(s) and the source, provide a link to the Creative Commons license, and indicate if changes were made. 
logarithmic potentials of orthogonal polynomials appearing in the calculation of the position and momentum information entropies of quantum systems (see Dehesa et al. [1] and Tcheutia [2]).

The linearization problem is of both old and recent interest. For example, a very long time ago, Ferrers [3] and Adams [4] have found the linearization formula for the Legendre polynomials $P_{n}(x)$. This linearization formula was generalized by Hylleraas [5]. He obtained Dougall's linearization formula for the ultraspherical polynomials. The basic idea behind obtaining such formula is obtaining a recurrence relation satisfied by the linearization coefficients, and then solving it. The standard linearization problem associated to Jacobi polynomials and to establish the conditions of nonnegativity of the linearization coefficients has been extensively studied by many authors (see, for instance, Askey and Gasper [6], Gasper [7, 8], Rahman [9], and Chaggara and Koepf [10]). The linearization coefficients are often expressed in terms of certain hypergeometric functions. Recently, Doha and Abd-Elhameed in [11] have established new linearization formulas for the products of the two nonsymmetric classes of Jacobi polynomials called 'Chebyshev polynomials of the third and fourth kinds'. Also, Abd-Elhameed in [12] and Abd-Elhameed et al. in [13] have introduced new product and linearization formulas for Jacobi polynomials of certain parameters. Moreover, Tcheutia [2] has discussed, in his interesting PhD thesis, the problems of connection, linearization, and duplication coefficients of classical orthogonal polynomials. There are some other articles concerning the connection and linearization formulas for various orthogonal polynomials (see, for instance, [14-21]).

The main objective of this article is to establish new linearization formulas for the product of two nonsymmetric Jacobi polynomials. Another objective is to introduce some applications based on the developed linearization formulas and their related hypergeometric functions. As far as we know, all the derived and deduced formulas are completely new and cannot be found in the literature.

The rest of the paper is organized as follows. The next section is devoted to presenting some properties of the classical Jacobi polynomials. In Section 3, some new reduction formulas for the linearization coefficients of some Jacobi polynomials are derived with the aid of employing some symbolic algebraic computation such as the algorithms of Zeilberger, Petkovsek and van Hoeij, hence four new linearization formulas for products of some nonsymmetric Jacobi polynomials are also given in this section. Finally, Section 4 is devoted to presenting two applications to the developed linearization formulas and their related hypergeometric functions.

\section{Some properties of classical Jacobi polynomials}

The classical Jacobi polynomials associated with the real parameters $(\gamma>-1, \delta>-1)$ (see Abramowich and Stegun [22], Andrews et al. [23] and Rainville [24]) are a sequence of polynomials $P_{n}^{(\gamma, \delta)}(x), x \in[-1,1](n=0,1,2, \ldots)$, each respectively of degree $n$. For our present purposes, it is more convenient to use the normalized Jacobi polynomials which were introduced by Rahman [9] and used in [25],

$$
R_{n}^{(\gamma, \delta)}(x)=\frac{P_{n}^{(\gamma, \delta)}(x)}{P_{n}^{(\gamma, \delta)}(1)}=\frac{n !}{(\gamma+1)_{n}} P_{n}^{(\gamma, \delta)}(x)={ }_{2} F_{1}\left(\begin{array}{c|c}
-n, n+\gamma+\delta+1 & \frac{1-x}{2} \\
\gamma+1
\end{array}\right),
$$


which satisfy the following orthogonality relation:

$$
\int_{-1}^{1}(1-x)^{\gamma}(1+x)^{\delta} R_{m}^{(\gamma, \delta)}(x) R_{n}^{(\gamma, \delta)}(x) d x= \begin{cases}0, & m \neq n \\ h_{n}^{(\gamma, \delta)}, & m=n,\end{cases}
$$

where

$$
h_{n}^{(\gamma, \delta)}=\frac{2^{\gamma+\delta+1} n ! \Gamma(n+\delta+1)[\Gamma(\gamma+1)]^{2}}{(2 n+\gamma+\delta+1) \Gamma(n+\gamma+\delta+1) \Gamma(n+\gamma+1)} .
$$

The definition in (3) enables one to write the six special polynomials of the general Jacobi polynomials as follows:

$$
\begin{aligned}
& C_{n}^{(\alpha)}(x)=R_{n}^{\left(\alpha-\frac{1}{2}, \alpha-\frac{1}{2}\right)}(x), \\
& T_{n}(x)=R_{n}^{\left(-\frac{1}{2},-\frac{1}{2}\right)}(x), \\
& U_{n}(x)=(n+1) R_{n}^{\left(\frac{1}{2}, \frac{1}{2}\right)}(x), \\
& V_{n}(x)=R_{n}^{\left(-\frac{1}{2}, \frac{1}{2}\right)}(x), \\
& W_{n}(x)=(2 n+1) R_{n}^{\left(\frac{1}{2},-\frac{1}{2}\right)}(x), \\
& P_{n}(x)=R_{n}^{(0,0)}(x),
\end{aligned}
$$

where $C_{n}^{(\alpha)}(x), T_{n}(x), U_{n}(x), V_{n}(x), W_{n}(x)$, and $P_{n}(x)$ are the ultraspherical polynomials, Chebyshev polynomials of the first, second, third, and fourth kinds, and Legendre polynomials, respectively.

The following identity is also of interest:

$$
R_{n}^{(\gamma, \delta)}(-x)=\frac{(-1)^{n} \Gamma(\gamma+1) \Gamma(n+\delta+1)}{\Gamma(\delta+1) \Gamma(n+\gamma+1)} R_{n}^{(\delta, \gamma)}(x) .
$$

For more properties on Jacobi polynomials and their special polynomials, one is referred to the important books of Andrews et al. [23] and Mason and Handscomb [26].

\section{Linearization formulas for the product of some nonsymmetric Jacobi polynomials}

This section is devoted to establishing new linearization formulas for some products of Jacobi polynomials of certain parameters. The basic idea behind obtaining the new derived formulas is based on reducing the linearization coefficients of Jacobi polynomials which were obtained by Rahman [9] with the aid of employing some symbolic algebraic algorithms such as the algorithms of Zeilberger, Petkovsek and van Hoeij.

\subsection{Linearization formulas for Jacobi polynomials}

There are several formulas linearizing the products of Jacobi polynomials. The authors in

[10] discussed the linearization problem

$$
P_{i}^{(\lambda, \delta)}(x) P_{j}^{(\mu, \gamma)}(x)=\sum_{k=0}^{i+j} L_{i j}(k) P_{k}^{(\alpha, \beta)}(x),
$$


and they proved that the linearization coefficients $L_{i j}(k)$ can be given explicitly by the formula

$$
\begin{aligned}
L_{i j}(i+j-k)= & \frac{(\alpha+1)_{i+j}(\alpha+\beta+2(i+j-k)+1)(\alpha+\beta+1)_{i+j-k}}{(\alpha+1)_{i+j-k}(\alpha+\beta+1)_{2(i+j)-k+1}} \\
& \times \frac{(-1)^{k}(i+j) !(\delta+\lambda+1)_{2 i}(\gamma+\mu+1)_{2 j}}{i ! j ! k !(\delta+\lambda+1)_{i}(\gamma+\mu+1)_{j}} \\
& \times F_{2: 1}^{2: 2}\left(\begin{array}{c}
-k,-\alpha-\beta-2(i+j)+k-1:-i,-\lambda-i ;-j ;-\mu-j ; \\
-(i+j),-\alpha-(i+j):-2 i-\lambda-\delta ;-2 j-\mu-\gamma ;
\end{array}\right),
\end{aligned}
$$

where the notation $F_{2: 1}^{2: 2}$ denotes the Kampé de Fériet function, which is represented as a double hypergeometric function. With respect to the coefficients $L_{i j}(k)$, it is not easy to give reduction formulas for them even for special choices of the involved parameters in (8). Moreover, the authors in [10] used the Gasper reduction formula in [27] for establishing the following new expression for the linearization coefficients for the case corresponding to the choices $\alpha=\mu+\lambda, \beta=\delta+\gamma$ :

$$
\begin{aligned}
L_{i j}(i+j-k)= & \frac{(\alpha+1)_{i+j}(\alpha+\beta+2(i+j-k)+1)(\alpha+\beta+1)_{i+j-k}}{(\alpha+1)_{i+j-k}(\alpha+\beta+1)_{2(i+j)-k+1}} \\
& \times \frac{(i+j) !(\delta+\lambda+1)_{2 i}(\gamma+\mu+1)_{2 j}(-2 i-\delta-\lambda)_{k}}{i ! j ! k !(\delta+\lambda+1)_{i}(\gamma+\mu+1)_{j}(-2 j-\mu-\gamma)_{k}} \\
& \times{ }_{3} F_{2}\left(\begin{array}{c}
-k,-i,-\gamma-\delta-2 i-2 j+k-\lambda-\mu-1 \\
-i-j,-\delta-2 i-\lambda
\end{array}\right) \\
& \times{ }_{3} F_{2}\left(\begin{array}{c}
-k,-i-\lambda,-\gamma-\delta-2 i-2 j+k-\lambda-\mu-1 \\
-\delta-2 i-\lambda,-i-j-\lambda-\mu
\end{array}\right) .
\end{aligned}
$$

Also, Rahman in [9] has developed another linearization formula for the Jacobi polynomials. This formula is expressed in terms of a terminating hypergeometric function of the type ${ }_{4} F_{3}(1)$, and it is explicitly stated in the following theorem.

Theorem 1 For all $j \geq i$, the following linearization formula holds:

$$
R_{i}^{(\gamma, \delta)}(x) R_{j}^{(\gamma, \delta)}(x)=\sum_{\ell=0}^{2 i} L_{i, j, \ell} R_{j-i+\ell}^{(\gamma, \delta)}(x),
$$

where

$$
\begin{aligned}
L_{i, j, \ell}= & \frac{j ! \Gamma(\gamma+1)(2 j-2 i+2 \ell+\gamma+\delta+1)}{(j-i) ! \ell ! \Gamma(2 j+\gamma+\delta+2)} \\
& \times \frac{\Gamma(j-i+\ell+\gamma+1) \Gamma(\delta+j+1) \Gamma(2 i+\gamma+\delta+1) \Gamma(2 j-2 i+\ell+\gamma+\delta+1)}{\Gamma(j-i+\ell+\delta+1) \Gamma(i+\gamma+\delta+1) \Gamma(\gamma+j-i+1) \Gamma(\gamma+i+1)} \\
& \times \sum_{r=0}^{\ell} \frac{(-\ell)_{r}(\ell+2 j-2 i+\gamma+\delta+1)_{r}}{r !(2 j+\gamma+\delta+2)_{r}} \\
& \times{ }_{4} F_{3}\left(\begin{array}{c}
-r, r+2 j-2 i+1,-i,-i-\delta \\
j-i+1, j-i+\gamma+1,-2 i-\gamma-\delta
\end{array}\right.
\end{aligned}
$$


It is worthy to note here that although the hypergeometric function ${ }_{4} F_{3}(1)$ that appears in (10) is balanced, it cannot be summed in a closed form except for special choices of its parameters. Doha and Abd-Elhameed in [11] have developed two reduction formulas for the ${ }_{4} F_{3}(1)$ in (10) for the two cases corresponding to $\gamma=-\delta=\frac{1}{2}$ and $\gamma=-\delta=\frac{-1}{2}$, and consequently they deduced two new linearization formulas for products of Chebyshev polynomials of third and fourth kinds. In this paper, we aim to give some new linearization formulas for products of some nonsymmetric Jacobi polynomials for certain choices of their parameters. In fact, our strategy to derive such formulas can be summarized in the following items:

1. Finding a closed form for the hypergeometric function which appears in (10). This can be performed through the following two stages:

- Deducing the recurrence relation satisfied by the terminating ${ }_{4} F_{3}(1)$ in $(10)$. For this purpose, the celebrated algorithm of Zeilberger (see, for instance, Koepf [28]) can be employed. More precisely, Maple software is used via the 'sumrecursion command'.

- Solving the resulted recurrence relation via any suitable computer algebra algorithm. The algorithm of Petkovsek (see Koepf [28]), or the improved one of van Hoeij [29], can be used. In addition, the package in Maple software called 'LREtools[hypergeomsols]' may be used for this purpose.

2. Substituting the ${ }_{4} F_{3}(1)$ by its reduced form in (10). The resulting sum is reduced by following similar procedures to the ones mentioned in the first item.

3. Performing some algebraic computation in order to obtain the desired linearization coefficients in a simple form, and hence the linearization formulas can be obtained.

In this respect, the linearization coefficients $L_{i, j, \ell}$ in (10) can be given in reduced forms for the special choices of $\alpha$ and $\beta$. In the following, we will show that $L_{i, j, \ell}$ can be written in a reduced form for the four cases corresponding to the following choices:
(i) $\quad \gamma=\frac{-1}{2}, \quad \delta=\frac{3}{2}$,
(ii) $\quad \gamma=\frac{1}{2}, \quad \delta=\frac{3}{2}$,
(iii) $\quad \gamma=\frac{3}{2}, \quad \delta=\frac{-1}{2}$,
(iv) $\gamma=\frac{3}{2}, \quad \delta=\frac{1}{2}$.

3.2 Linearization formula for the product $R_{i}^{\left(\frac{-1}{2}, \frac{3}{2}\right)}(x) R_{j}^{\left(\frac{-1}{2}, \frac{3}{2}\right)}(x)$

In order to proceed according to the strategy explained in Section 3.1, the following two lemmas are essential.

Lemma 1 For all $r, i, j \in \mathbb{Z}^{\geq 0}$ and $r \leq 2 i+1$, one has

$$
\begin{aligned}
{ }_{4} F_{3} & \left(\begin{array}{c}
-r, r+2 j-2 i+1,-i,-i-\frac{3}{2} \\
j-i+1, j-i+\frac{1}{2},-2 i-1
\end{array} \mid 1\right) \\
& =\frac{(i(4 j+6)+2 j-2 r+3)(2 j-2 i) !(2 j+r+2) !}{(2 i+1)(2 j+3) !(2 j-2 i+r) !} .
\end{aligned}
$$

Proof First, we set

$$
{ }_{4} F_{3}\left(\begin{array}{c|c}
-r, r+2 j-2 i+1,-i,-i-\frac{3}{2} \\
j-i+1, j-i+\frac{1}{2},-2 i-1
\end{array} \mid 1\right)=A_{i, j, r}
$$


then making use of the celebrated algorithm of Zeilberger (see, for instance, Koepf [28]), and in particular via the 'sumrecursion command' in Maple software, it can be shown that the following recurrence relation of order two is satisfied by $A_{i, j, r}$ :

$$
\begin{aligned}
& (r-1)(2 j+r+1) A_{i, j, r-2}+\left(4 i j+4 r i-4 j r-2 r^{2}+2 i+6 j+2 r+1\right) A_{i, j, r-1} \\
& \quad+(2 i-2 j-r)(-r+2+2 i) A_{i, j, r}=0
\end{aligned}
$$

with the initial values

$$
A_{i, j, 0}=1, \quad A_{i, j, 1}=\frac{-(4 i j+6 i+2 j+1)}{(1+2 i)(-1+2 i-2 j)} .
$$

This recurrence relation can be explicitly solved through Petkovsek's algorithm (see Koepf [28]), or through the improved version of van Hoeij [29], to give

$$
A_{i, j, r}=\frac{(i(4 j+6)+2 j-2 r+3)(2 j-2 i) !(2 j+r+2) !}{(2 i+1)(2 j+3) !(2 j-2 i+r) !} .
$$

Lemma $2 \forall j, r, \ell \in \mathbb{Z}^{\geq 0}$, one has

$$
\begin{aligned}
& \sum_{r=0}^{\ell} \frac{(i(4 j+6)+2 j-2 r+3)(-\ell)_{r}(-2 i+2 j+\ell+r+1) !}{r !(-2 i+2 j+r) !} \\
& \quad=(-1)^{\ell+1}(\ell+1) !(\ell-2 i-1)(2 j+\ell+3)(\ell-2 i+2 j+1) .
\end{aligned}
$$

Proof Let us denote

$$
\sum_{r=0}^{\ell} \frac{(i(4 j+6)+2 j-2 r+3)(-\ell)_{r}(-2 i+2 j+\ell+r+1) !}{r !(-2 i+2 j+r) !}=B_{i, j, \ell} .
$$

If Zeilberger's algorithm is employed, then one can see that $B_{i, j, \ell}$ satisfies the following recurrence relation of order one:

$$
\begin{gathered}
(\ell+1)(\ell+2 j+3)(\ell-2 i-1)(1-2 i+2 j+\ell) B_{i, j, \ell-1} \\
+(\ell+2 j+2)(\ell-2 i-2)(-2 i+2 j+\ell) B_{i, j, \ell}=0,
\end{gathered}
$$

with the initial value

$$
B_{i, j, 0}=-(4 i j+6 i+2 j+3)(-1+2 i-2 j),
$$

which can be immediately solved to give

$$
B_{i, j, \ell}=(-1)^{\ell+1}(\ell+1) !(\ell-2 i-1)(2 j+\ell+3)(\ell-2 i+2 j+1) .
$$

Now, based on Theorem 1 and Lemmas 1 and 2, we give the following linearization formula for the product $R_{i}^{\left(\frac{-1}{2}, \frac{3}{2}\right)}(x) R_{j}^{\left(\frac{-1}{2}, \frac{3}{2}\right)}(x)$. 
Theorem 2 For all nonnegative integers $i, j$ and $j \geq i$, the following linearization formula holds:

$$
\begin{aligned}
& R_{i}^{\left(-\frac{1}{2}, \frac{3}{2}\right)}(x) R_{j}^{\left(-\frac{1}{2}, \frac{3}{2}\right)}(x) \\
& =\frac{1}{(i+1)(j+1)} \sum_{\ell=0}^{2 i} \frac{(-1)^{\ell+1}(\ell+1)(\ell-2 i-1)(\ell+2 j+3)(\ell-i+j+1)(\ell-2 i+2 j+1)}{(2 \ell-2 i+2 j+1)(2 \ell-2 i+2 j+3)} \\
& \quad \times R_{j-i+\ell}^{\left(-\frac{1}{2}, \frac{3}{2}\right)}(x) .
\end{aligned}
$$

Proof If we substitute $\gamma=-\frac{1}{2}$ and $\delta=\frac{3}{2}$ into (9), then we get

$$
R_{i}^{\left(-\frac{1}{2}, \frac{3}{2}\right)}(x) R_{j}^{\left(-\frac{1}{2}, \frac{3}{2}\right)}(x)=\sum_{\ell=0}^{2 i} L_{i, j, \ell} R_{j-i+\ell}^{\left(-\frac{1}{2}, \frac{3}{2}\right)}(x), \quad j \geq i
$$

where

$$
\begin{aligned}
L_{i, j, \ell}= & \frac{(2 i+1)(2 j+3)(-i+j+\ell+1)(-2 i+2 j+\ell+1) !}{(i+1)(j+1) \ell !(2 i-2 j-2 \ell-3)(2 i-2 j-2 \ell-1)(2 j-2 i) !} \\
& \times \sum_{r=0}^{\ell} \frac{(-\ell)_{r}(-2 i+2 j+\ell+2)_{r}}{r !(2 j+3)_{r}}{ }_{4} F_{3}\left(\begin{array}{c}
-r, r+2 j-2 i+1,-i,-i-\frac{3}{2} \\
j-i+1, j-i+\frac{1}{2},-2 i-1
\end{array} \mid 1\right) .
\end{aligned}
$$

Now, the application of Lemma 1 enables one to express explicitly the linearization coefficients $L_{i, j, \ell}$ as

$$
\begin{aligned}
L_{i, j, \ell}= & \frac{(2 i+1)(2 j+3)(-i+j+\ell+1)(-2 i+2 j+\ell+1) !}{(i+1)(j+1) \ell !(2 i-2 j-2 \ell-3)(2 i-2 j-2 \ell-1)(2 j-2 i) !} \\
& \times \sum_{r=0}^{\ell} \frac{(-\ell)_{r}(-2 i+2 j+\ell+2)_{r}}{r !(2 j+3)_{r}} \frac{(i(4 j+6)+2 j-2 r+3)(2 j-2 i) !(2 j+r+2) !}{(2 i+1)(2 j+3) !(2 j-2 i+r) !},
\end{aligned}
$$

which can be simplified again to take the form

$$
\begin{aligned}
L_{i, j, \ell}= & \frac{(-i+j+\ell+1)}{(i+1)(j+1) \ell !(-2 i+2 j+2 \ell+1)(-2 i+2 j+2 \ell+3)} \\
& \times \sum_{r=0}^{\ell} \frac{(i(4 j+6)+2 j-2 r+3)(-\ell)_{r}(-2 i+2 j+\ell+r+1) !}{r !(-2 i+2 j+r) !} .
\end{aligned}
$$

Now, and with the aid of Lemma 2, the linearization formula (15) can be written in the following reduced form:

$$
\begin{aligned}
& R_{i}^{\left(-\frac{1}{2}, \frac{3}{2}\right)}(x) R_{j}^{\left(-\frac{1}{2}, \frac{3}{2}\right)}(x) \\
& =\frac{1}{(i+1)(j+1)} \sum_{\ell=0}^{2 i} \frac{(-1)^{\ell+1}(\ell+1)(\ell-2 i-1)(\ell+2 j+3)(\ell-i+j+1)(\ell-2 i+2 j+1)}{(2 \ell-2 i+2 j+1)(2 \ell-2 i+2 j+3)} \\
& \quad \times R_{j-i+\ell}^{\left(-\frac{1}{2}, \frac{3}{2}\right)}(x),
\end{aligned}
$$

and therefore the proof of Theorem 2 is completed. 


\subsection{Linearization formula for the product $R_{i}^{\left(\frac{1}{2}, \frac{3}{2}\right)}(x) R_{j}^{\left(\frac{1}{2}, \frac{3}{2}\right)}(x)$}

In this section, we follow the steps explained in the strategy mentioned in Section 3.1. In detail, we show that the ${ }_{4} F_{3}(1)$ which appears in (10) can be reduced for the choice corresponding to $\gamma=\frac{1}{2}, \delta=\frac{3}{2}$. Hence, a simple linearization formula for the product $R_{i}^{\left(\frac{1}{2}, \frac{3}{2}\right)}(x) R_{j}^{\left(\frac{1}{2}, \frac{3}{2}\right)}(x)$ can be obtained. For this purpose, the following two lemmas are needed.

Lemma 3 For all $r, i, j \in \mathbb{Z}^{\geq 0}$ and $r \leq 2 i+2$, one has

$$
\begin{aligned}
{ }_{4} F_{3} & \left(\begin{array}{c}
-r, r+2 j-2 i+1,-i,-i-\frac{3}{2} \\
j-i+1, j-i+\frac{3}{2},-2 i-2
\end{array} \mid 1\right) \\
= & \frac{(r-2(i+1)(j+2))(2 j-2 i+1) !(2 j+r+3) !}{(i+1)(2 j+4) !(2 i-2 j-2 r-1)(2 j-2 i+r) !} .
\end{aligned}
$$

Proof If we set

$$
{ }_{4} F_{3}\left(\begin{array}{c}
-r, r+2 j-2 i+1,-i,-i-\frac{3}{2} \\
j-i+1, j-i+\frac{3}{2},-2 i-2
\end{array} \mid 1\right)=S_{i, j, r},
$$

then with the aid Zeilberger's algorithm, $S_{i, j, r}$, satisfies the following recurrence relation:

$$
\begin{aligned}
& (r-1)(r+2 j+2)(2 i-2 j-2 r+3) S_{i, j, r-2}+2\left(2 i j+2 r i-2 j r-r^{2}+2 i+4 j+r+3\right) \\
& \quad \times(-2 r-2 j+2 i+1) S_{i, j, r-1}+(-r-2 j+2 i)(-r+2 i+3)(-2 r-2 j+2 i-1) S_{i, j, r} \\
& \quad=0
\end{aligned}
$$

with the initial values

$$
S_{i, j, 0}=1, \quad S_{i, j, 1}=\frac{-(2 i j+4 i+2 j+3)}{(1+i)(-2 j+2 i-3)},
$$

which can be solved exactly with the aid of the any suitable computer algebra algorithm such the algorithms of Petkovsek and van Hoeij (see Koepf [28] and van Hoeij [29]), to give

$$
S_{i, j, r}=\frac{(r-2(i+1)(j+2))(2 j-2 i+1) !(2 j+r+3) !}{(i+1)(2 j+4) !(2 i-2 j-2 r-1)(2 j-2 i+r) !} .
$$

Lemma $4 \forall j, r, \ell \in \mathbb{Z}^{\geq 0}$, one has

$$
\begin{aligned}
\sum_{r=0}^{\ell} & \frac{(2 i j+4 i+2 j-r+4)(-\ell)_{r}(-2 i+2 j+\ell+3)_{r}}{r !(-2 i+2 j+2 r+1)(-2 i+2 j+r) !} \\
& = \begin{cases}\frac{(\ell+2) !(2 i-\ell+2)(2 j+\ell+4)}{4(\ell+1)(-2 i+2 j+\ell+1) !}, & \text { if } \ell \text { even, }, \\
\frac{(\ell+1) !(-2 i+\ell-1)(2 j+\ell+5)}{4(-2 i+2 j+\ell+2)(-2 i+2 j+\ell) !}, & \text { if } \ell \text { odd. }\end{cases}
\end{aligned}
$$

Theorem 3 For all nonnegative integers $i, j$ and $j \geq i$, the following linearization formula holds:

$$
R_{i}^{\left(\frac{1}{2}, \frac{3}{2}\right)}(x) R_{j}^{\left(\frac{1}{2}, \frac{3}{2}\right)}(x)=\sum_{\ell=0}^{2 i} L_{i, j, \ell} R_{j-i+\ell}^{\left(\frac{1}{2}, \frac{3}{2}\right)}(x),
$$


where

$$
\begin{aligned}
L_{i, j, \ell}= & \frac{1}{8(i+1)(i+2)(j+1)(j+2)} \\
& \times \begin{cases}(\ell+2)(\ell-2 i-2)(2 j+\ell+4)(2 i-2 j-\ell-2), & \text { if } \ell \text { even }, \\
(\ell+1)(2 i-\ell+1)(2 j+\ell+5)(2 i-2 j-\ell-1), & \text { if } \ell \text { odd } .\end{cases}
\end{aligned}
$$

Proof If we substitute by $\gamma=\frac{1}{2}$ and $\delta=\frac{3}{2}$ into relation (9), then we get

$$
R_{i}^{\left(\frac{1}{2}, \frac{3}{2}\right)}(x) R_{j}^{\left(\frac{1}{2}, \frac{3}{2}\right)}(x)=\sum_{\ell=0}^{2 i} L_{i, j, \ell} R_{j-i+\ell}^{\left(\frac{1}{2}, \frac{3}{2}\right)}(x), \quad j \geq i,
$$

where

$$
\begin{aligned}
L_{i, j, \ell}= & \frac{(-2 i+2 j+\ell+2) !}{(i+2)(j+1) \ell !(-2 i+2 j+1) !} \\
& \times \sum_{r=0}^{\ell} \frac{(-\ell)_{r}(-2 i+2 j+\ell+3)_{r}}{r !(2 j+4)_{r}}{ }_{4} F_{3}\left(\begin{array}{c}
-r, r+2 j-2 i+1,-i,-i-\frac{3}{2} \\
j-i+1, j-i+\frac{3}{2},-2 i-2
\end{array} \mid 1\right) .
\end{aligned}
$$

Making use of Lemma 3 enables one to reduce the linearization coefficients $L_{i, j, \ell}$ in (22) to take the form

$$
\begin{aligned}
L_{i, j, \ell}= & \frac{(-2 i+2 j+\ell+2) !}{2(i+1)(i+2)(j+1)(j+2) \ell !} \\
& \times \sum_{r=0}^{\ell} \frac{(2 i j+4 i+2 j-r+4)(-\ell)_{r}(-2 i+2 j+\ell+3)_{r}}{r !(-2 i+2 j+2 r+1)(-2 i+2 j+r) !},
\end{aligned}
$$

which can be converted with the application of Lemma 4 into the more simplified form

$$
\begin{aligned}
L_{i, j, \ell}= & \frac{1}{8(i+1)(i+2)(j+1)(j+2)} \\
& \times \begin{cases}(\ell+2)(\ell-2 i-2)(2 j+\ell+4)(2 i-2 j-\ell-2), & \text { if } \ell \text { even, } \\
(\ell+1)(2 i-\ell+1)(2 j+\ell+5)(2 i-2 j-\ell-1), & \text { if } \ell \text { odd },\end{cases}
\end{aligned}
$$

and this completes the proof of Theorem 3.

\subsection{Other two linearization formulas for some Jacobi polynomials}

Based on relation (6), and if $x$ is replaced by $(-x)$ in the two linearization formulas (14) and (20), the following two linearization formulas can easily be obtained:

$$
\begin{aligned}
R_{i}^{\left(\frac{3}{2}, \frac{-1}{2}\right)}(x) R_{j}^{\left(\frac{3}{2}, \frac{-1}{2}\right)}(x) & \\
= & \frac{3}{(i+1)(2 i+1)(2 i+3)(j+1)(2 j+1)(2 j+3)} \\
& \quad \times \sum_{\ell=0}^{2 i}(\ell+1)(2 i-\ell+1)(2 j+\ell+3)(2 i-2 j-\ell-1)(i-j-\ell-1) R_{j-i+\ell}^{\left(\frac{3}{2}, \frac{-1}{2}\right)}(x),
\end{aligned}
$$


and

$$
R_{i}^{\left(\frac{3}{2}, \frac{1}{2}\right)}(x) R_{j}^{\left(\frac{3}{2}, \frac{1}{2}\right)}(x)=\sum_{\ell=0}^{2 i} \bar{L}_{i, j, \ell} R_{j-i+\ell}^{\left(\frac{3}{2}, \frac{1}{2}\right)}(x)
$$

where

$$
\begin{aligned}
\bar{L}_{i, j, \ell}= & \frac{3(-1)^{\ell}}{8(i+1)(2 i+1)(2 i+3)(j+1)(2 j+1)(2 j+3)} \\
& \times \begin{cases}(\ell+2)(2 i-\ell+2)(2 j+\ell+4)(2 i-2 j-2 \ell-3)(2 i-2 j-\ell-2), & \text { if } \ell \text { even, } \\
(\ell+1)(\ell-2 i-1)(2 j+\ell+5)(2 i-2 j-2 \ell-3)(2 i-2 j-\ell-1), & \text { if } \ell \text { odd }\end{cases}
\end{aligned}
$$

\section{Two applications to the derived linearization formulas and their related hypergeometric functions}

In this section we present two applications to the developed linearization formulas and their related hypergeometric functions. In the first application, four new formulas for definite integrals involving three products of Jacobi polynomials of certain parameters are given, while in the second, and based on a certain Whipple transformation, two new reduction formulas for certain hypergeometric functions of the type ${ }_{4} F_{3}(1)$ are deduced.

\subsection{New closed formulas for four definite integrals}

In this subsection, and based on the four developed linearization formulas (14), (20), (25), and (26), new formulas for four integrals involving products of nonsymmetric Jacobi polynomials can be deduced. The results are given in the following two corollaries.

Corollary 1 For all $j-i \leq m$, one has

$$
\begin{aligned}
& \int_{-1}^{1} \frac{(x+1)^{3 / 2}}{\sqrt{1-x}} R_{i}^{\left(\frac{-1}{2}, \frac{3}{2}\right)}(x) R_{j}^{\left(\frac{-1}{2}, \frac{3}{2}\right)}(x) R_{m}^{\left(\frac{-1}{2}, \frac{3}{2}\right)}(x) d x \\
& =\frac{\pi(-1)^{i+j+m+1}(i-j-m-1)(i+j-m+1)(i+j+m+1)(i+j+m+3)}{2(i+1)(j+1)(m+1)}, \\
& \int_{-1}^{1} \sqrt{1-x}(x+1)^{3 / 2} R_{i}^{\left(\frac{1}{2}, \frac{3}{2}\right)}(x) R_{j}^{\left(\frac{1}{2}, \frac{3}{2}\right)}(x) R_{m}^{\left(\frac{1}{2}, \frac{3}{2}\right)}(x) d x \\
& =\frac{\pi}{8(i+1)(i+2)(j+1)(j+2)(m+1)(m+2)}
\end{aligned}
$$

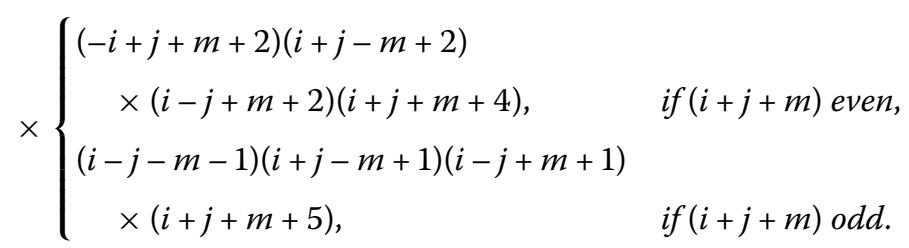

Corollary 2 For all $j-i \leq m$, one has

$$
\begin{aligned}
& \int_{-1}^{1} \frac{(1-x)^{3 / 2}}{\sqrt{x+1}} R_{i}^{\left(\frac{3}{2}, \frac{-1}{2}\right)}(x) R_{j}^{\left(\frac{3}{2}, \frac{-1}{2}\right)}(x) R_{m}^{\left(\frac{3}{2}, \frac{-1}{2}\right)}(x) d x \\
& =\frac{27 \pi(-i+j+m+1)(i+j-m+1)(i-j+m+1)(i+j+m+3)}{2(i+1)(2 i+1)(2 i+3)(j+1)(2 j+1)(2 j+3)(m+1)(2 m+1)(2 m+3)},
\end{aligned}
$$




$$
\begin{aligned}
& \int_{-1}^{1}(1-x)^{3 / 2} \sqrt{x+1} R_{i}^{\left(\frac{3}{2}, \frac{1}{2}\right)}(x) R_{j}^{\left(\frac{3}{2}, \frac{1}{2}\right)}(x) R_{m}^{\left(\frac{3}{2}, \frac{1}{2}\right)}(x) d x \\
& =\frac{27 \pi(-1)^{i+j+m}}{8(i+1)(i+2)(2 i+3)(j+1)(j+2)(2 j+3)(m+1)(m+2)(2 m+3)}
\end{aligned}
$$

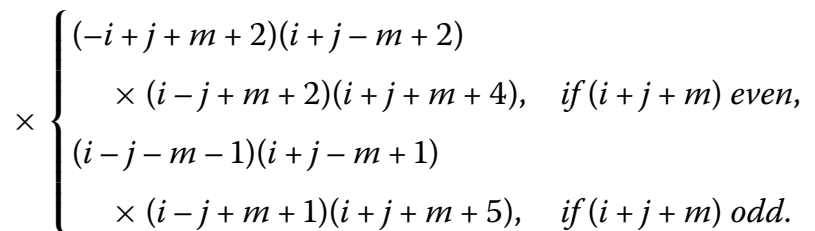

Proof First consider the linearization problem

$$
R_{i}^{(\gamma, \delta)}(x) R_{j}^{(\gamma, \delta)}(x)=\sum_{\ell=0}^{2 i} L_{i, j, \ell} R_{j-i+\ell}^{(\gamma, \delta)}(x) .
$$

If we multiply both sides of (31) by $(1-x)^{\gamma}(1+x)^{\delta} R_{m}^{(\gamma, \delta)}(x)$, and integrate over the interval $(-1,1)$, then we get

$$
\begin{aligned}
& \int_{-1}^{-1}(1-x)^{\gamma}(1+x)^{\delta} R_{i}^{(\gamma, \delta)}(x) R_{j}^{(\gamma, \delta)}(x) R_{m}^{(\gamma, \delta)}(x) d x \\
& =\sum_{\ell=0}^{2 i} L_{i, j, \ell} \int_{-1}^{1}(1-x)^{\gamma}(1+x)^{\delta} R_{m}^{(\gamma, \delta)}(x) R_{j-i+\ell}^{(\gamma, \delta)}(x) d x .
\end{aligned}
$$

Making use of the orthogonality relation (4), it is easy to show that

$$
\int_{-1}^{-1}(1-x)^{\gamma}(1+x)^{\delta} R_{i}^{(\gamma, \delta)}(x) R_{j}^{(\gamma, \delta)}(x) R_{m}^{(\gamma, \delta)}(x) d x=L_{i, j, m+i-j} h_{m}^{(\gamma, \delta)},
$$

where the linearization coefficients $L_{i, j, \ell}$ are as given in (10), and $h_{m}^{(\gamma, \delta)}$ is given by (5). The results of Corollaries 1 and 2 can be obtained if the linearization coefficients $L_{i, j, \ell}$ are substituted by their reduced forms for the four cases corresponding to the following choices:
(i) $\gamma=\frac{-1}{2}, \quad \delta=\frac{3}{2}$,
(ii) $\gamma=\frac{1}{2}, \quad \delta=\frac{3}{2}$,
(iii) $\gamma=\frac{3}{2}, \quad \delta=\frac{-1}{2}$,
(iv) $\gamma=\frac{3}{2}, \quad \delta=\frac{1}{2}$.

\subsection{Reduction formulas for certain hypergeometric functions of unit argument}

This subsection is interested in introducing two new reduction formulas for summing two terminating hypergeometric series. These reduction formulas can be obtained with the aid of Whipple's transformation stated in the following theorem.

Theorem 4 For any positive integer $r$ (Andrews et al. [23]), one has

$$
{ }_{4} F_{3}\left(\begin{array}{c}
-r, a, b, c \\
d, e, f
\end{array} \mid 1\right)=\frac{(e-a)_{r}(f-a)_{r}}{(e)_{r}(f)_{r}}{ }_{4} F_{3}\left(\begin{array}{c}
-r, a, d-b, d-c \\
d, a+1-r-e, a+1-r-f
\end{array} \mid 1\right)
$$

where $-r+a+b+c+1=d+e+f$. 
Now, we state and prove the following theorem.

Theorem 5 For any positive integer $r$, the following two reduction formulas hold:

$$
\begin{aligned}
{ }_{4} F_{3} & \left(\begin{array}{c}
-r, j+1, j+\frac{5}{2},-2 i+2 j+r+1 \\
-i+j+1,-i+j+\frac{3}{2}, 2 j+3
\end{array} \mid 1\right) \\
= & \frac{(2 j-2 i) !(2 j+r+2) !(4 i j+6 i+2 j-2 r+3)(-2 i-1)_{r}\left(-i+j+\frac{1}{2}\right)_{r}}{(2 i+1)(2 j+3) !(-2 j-r-2)_{r}(-2 i+2 j+r) !\left(i-j-r-\frac{1}{2}\right)_{r}}
\end{aligned}
$$

and

$$
\begin{aligned}
{ }_{4} F_{3} & \left(\begin{array}{c}
-r, j+1, j+\frac{5}{2},-2 i+2 j+r+1 \\
-i+j+1,-i+j+\frac{1}{2}, 2 j+4
\end{array} \mid 1\right) \\
& =\frac{(-2 i+2 j+1) !(2 j+r+3) !(-2 i j-4 i-2 j+r-4)(-2(i+1))_{r}\left(-i+j+\frac{3}{2}\right)_{r}}{(i+1)(2 j+4) !(2 i-2 j-2 r-1)(-2 j-r-3)_{r}(-2 i+2 j+r) !\left(i-j-r+\frac{1}{2}\right)_{r}} .
\end{aligned}
$$

Proof Making use of Whipple's transformation stated in Theorem 4 yields the following two transformation formulas:

$$
\begin{aligned}
{ }_{4} F_{3} & \left(\begin{array}{c}
-r, j+1, j+\frac{5}{2},-2 i+2 j+r+1 \\
-i+j+1,-i+j+\frac{3}{2}, 2 j+3
\end{array} \mid 1\right) \\
& =\frac{(-2 i-1)_{r}\left(-i+j+\frac{1}{2}\right)_{r}}{(-2 j-r-2)_{r}\left(i-j-r-\frac{1}{2}\right)_{r}}{ }_{4} F_{3}\left(\begin{array}{c}
-r, r+2 j-2 i+1,-i,-i-\frac{3}{2} \\
j-i+1, j-i+\frac{1}{2},-2 i-1
\end{array} \mid 1\right),
\end{aligned}
$$

and

$$
\begin{aligned}
& { }_{4} F_{3}\left(\begin{array}{c}
-r, j+1, j+\frac{5}{2},-2 i+2 j+r+1 \\
-i+j+\frac{1}{2},-i+j+1,2 j+4
\end{array} \mid 1\right) \\
& =\frac{(-2(i+1))_{r}\left(-i+j+\frac{3}{2}\right)_{r}}{(-2 j-r-3)_{r}\left(i-j-r+\frac{1}{2}\right)_{r}}{ }_{4} F_{3}\left(\begin{array}{c}
-r, r+2 j-2 i+1,-i,-i-\frac{3}{2} \\
j-i+1, j-i+\frac{3}{2},-2 i-2
\end{array} \mid 1\right) .
\end{aligned}
$$

The two reduction formulas (33) and (34) can be deduced from the application of Lemmas 1 and 3 on the right-hand sides of the two transformation formulas (35) and (36).

\section{Competing interests}

The author declares that he has no competing interests.

\section{Author's contributions}

The author declares that he carried out all the work in this manuscript and read and approved the final manuscript.

\section{Acknowledgements}

The author would like to thank the anonymous referees for their valuable and constructive comments, which improved the manuscript in its present form.

Received: 14 November 2014 Accepted: 17 May 2015 Published online: 04 June 2015

\section{References}

1. Dehesa, JS, Martínez-Finkelshtein, A, Sánchez-Ruiz, JS: Quantum information entropies and orthogonal polynomials. J. Comput. Appl. Math. 133, 23-46 (2001)

2. Tcheutia, DD: On Connection, Linearization and Duplication Coefficients of Classical Orthogonal Polynomials. PhD thesis, University of Kassel (2014). https://kobra.bibliothek.uni-kassel.de/handle/urn:nbn:de:hebis:34-2014071645714 
3. Ferrers, NM: An Elementary Treatise on Spherical Harmonics and Subjects Connected with Them. Macmillan \& Co., London (1877)

4. Adams, JC: On the expression of the product of any two Legendre's coefficients by means of a series of Legendre's coefficients. Proc. R. Soc. Lond. 27, 63-71 (1878)

5. Hylleraas, EA: Linearization of products of Jacobi polynomials. Math. Scand. 10, 189-200 (1962)

6. Askey, R, Gasper, G: Linearization of the product of Jacobi polynomials III. Can. J. Math. 23, 332-338 (1971)

7. Gasper, G: A linearization of the product of Jacobi polynomials I. Can. J. Math. 22, 172-175 (1970)

8. Gasper, G: A linearization of the product of Jacobi polynomials II. Can. J. Math. 22, 582-593 (1970)

9. Rahman, M: A non-negative representation of the linearization coefficients of the product of Jacobi polynomials. Can. J. Math. 33, 915-928 (1981)

10. Chaggara, H, Koepf, W: On linearization coefficients of Jacobi polynomials. Appl. Math. Lett. 23, 609-614 (2010)

11. Doha, EH, Abd-Elhameed, WM: New linearization formulae for the products of Chebyshev polynomials of third and fourth kind. Rocky Mt. J. Math. (2015). http://projecteuclid.org/euclid.rmjm/1405519304

12. Abd-Elhameed, WM: New product and linearization formulae of Jacobi polynomials of certain parameters. Integral Transforms Spec. Funct. (2015). doi:10.1080/10652469.2015.1029924

13. Abd-Elhameed, WM, Doha, EH, Ahmed, HM: Linearization formulae for certain Jacobi polynomials. Ramanujan J. (2015). doi:10.1007/s11139-014-9668-2

14. Maroni, $\mathrm{P}, \mathrm{da}$ Rocha, Z: Connection coefficients between orthogonal polynomials and the canonical sequence: an approach based on symbolic computation. Numer. Algorithms 47, 291-314 (2008)

15. Sánchez-Ruiz, J: Linearization and connection formulae involving squares of Gegenbauer polynomials. Appl. Math. Lett. 14, 261-267 (2001)

16. Sánchez-Ruiz, J, Artés, PL, Martínez-Finkelshtein, A, Dehesa, JS: General linearization formulae for products of continuous hypergeometric-type polynomials. J. Phys. A, Math. Gen. 32, 7345-7366 (1999)

17. Sánchez-Ruiz, J, Dehesa, JS: Some connection and linearization problems for polynomials in and beyond the Askey scheme. J. Comput. Appl. Math. 133, 579-591 (2001)

18. Area, I, Godoy, E, Ronveaux, A, Zarzo, A: Solving connection and linearization problems within the Askey scheme and its $q$-analogue via inversion formulas. J. Comput. Appl. Math. 136, 152-162 (2001)

19. Belmehdi, S, Lewanowicz, S, Ronveaux, A: Linearization of products of orthogonal polynomials of a discrete variable. Appl. Math. 24, 445-455 (1997)

20. Chaggara, $\mathrm{H}, \mathrm{Koepf}, \mathrm{W}$ : On linearization and connection coefficients for generalized Hermite polynomials. J. Comput. Appl. Math. 236, 65-73 (2011)

21. Foupouagnigni, M, Koepf, W, Tcheutia, DD: Connection and linearization coefficients of the Askey-Wilson polynomials. J. Symb. Comput. 53, 96-118 (2013)

22. Abramowitz, M, Stegun, IA (eds.): Handbook of Mathematical Functions. Applied Mathematical Series, vol. 55 National Bureau of Standards, New York (1970)

23. Andrews, GE, Askey, R, Roy, R: Special Functions. Cambridge University Press, Cambridge (1999)

24. Rainville, ED: Special Functions. Macmillan Co., New York (1960)

25. Doha, EH, Abd-Elhameed, WM, Ahmed, HM: The coefficients of differentiated expansions of double and triple Jacobi polynomials. Bull. Iran. Math. Soc. 38, 739-766 (2012)

26. Mason, JC, Handscomb, DC: Chebyshev Polynomials. Chapman \& Hall/CRC, Boca Raton (2003)

27. Gasper, G: Nonnegativity of a discrete Poisson kernel for the Hahn polynomials. J. Math. Anal. Appl. 42, 438-451 (1973)

28. Koepf, W: Hypergeometic Summation. Vieweg, Wiesbaden (1998)

29. van Hoeij, M: Finite singularities and hypergeometric solutions of linear recurrence equations. J. Pure Appl. Algebra 139, 109-131 (1998)

\section{Submit your manuscript to a SpringerOpen ${ }^{\circ}$ journal and benefit from:}

- Convenient online submission

Rigorous peer review

- Immediate publication on acceptance

- Open access: articles freely available online

- High visibility within the field

- Retaining the copyright to your article 